\title{
Pigmentverändungen vorbeugen
}

FotoUltra Spot Prevent bietet einen sehr hohen Schutz vor UV-Strahlen - der Hauptursache für Pigmentbildungen. Der sehr hohe Lichtschutzfaktor (SPF 50+) hilft Hautpigmentierungen vorzubeugen sowie auch die Bildung neuer sonnenbedingter Pigmentstörungen zu stoppen. Der enthaltene Lichtschutz ist 3-mal höher als der erforderliche Mindestschutz eines Sonnenschutzmittels.

\section{Verbesserung der Hautelastizität}

Durch die enthaltene Hyaluronsäure wird außerdem die Elastizität der Haut verbessert und Falten gemindert. Die Fusion-Fluid-Textur verschmilzt mit der Haut und ermöglicht so eine einfache und angenehme tägliche Anwendung. Das Fluid sollte eine halbe Stunde vor der Sonnenexposition großzügig auf die trockene Haut aufgetragen werden. Alle zwei Stunden sowie nach dem Schwitzen, Schwimmen oder Abtrocknen mit dem Handtuch wiederholen. Zur Vorbeugung von Pigmentstörungen sollte eine direkte Sonneneinstrahlung vermieden wer-

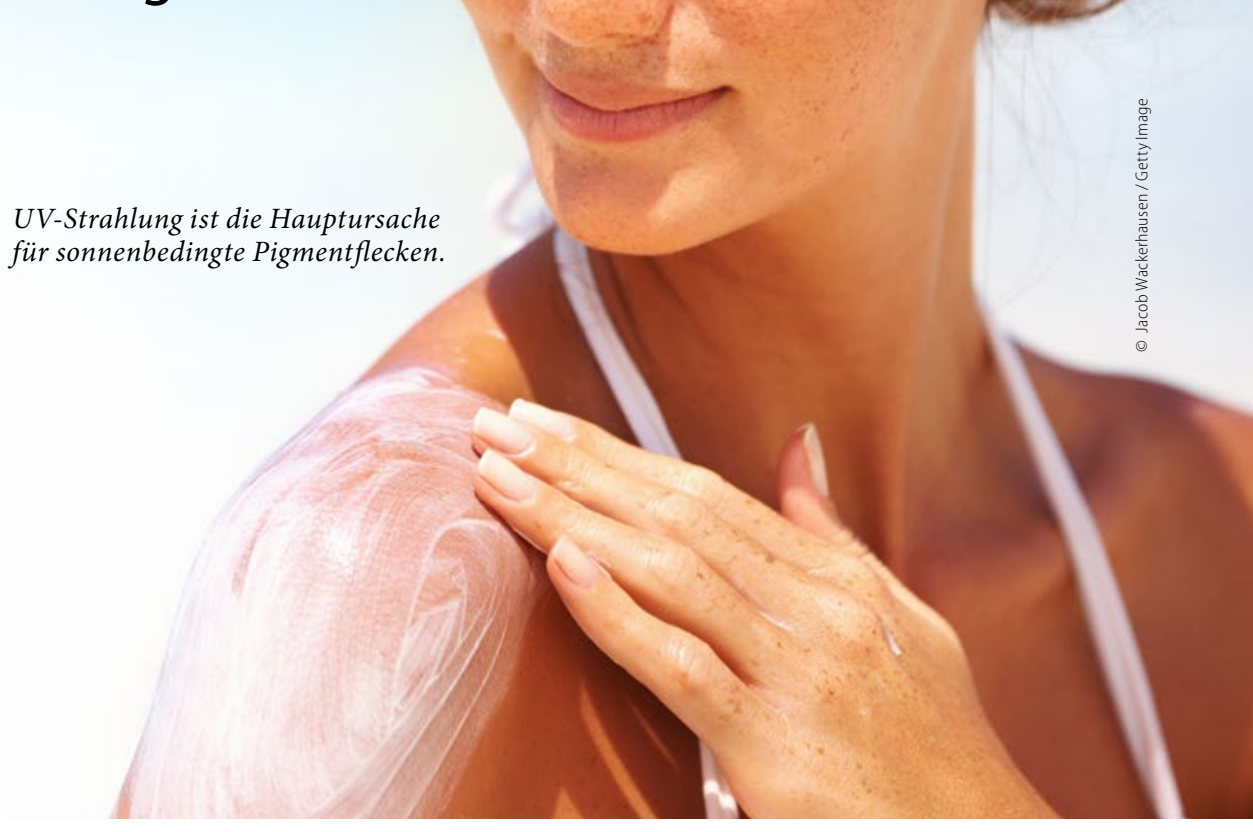

den. FotoUltra Spot Prevent ist eines von drei medizinischen Sonnenschutzprodukten aus der FotoUltra Reihe der Firma ISDIN: FotoUltra Solar Allergy zur Linderung der Symptome einer Sonnen- allergie sowie FotoUltra Active Unify zur Prävention und Reduktion von Pigmentflecken runden das Produktportfolio ab.

Nach Informationen von ISDIN

\section{Microfokussierter Ultraschal stimuliert Kollagenneogenese in der Tiefe}

\section{Nicht invasives Lifting}

Mit einer Kombination von mikrofokussiertem Ultraschall, Fillern und Botulinumtoxin können natürliche Ergebnisse bei der Gesichtsrejuvenation erreicht werden. Hierzu wurden aktuell auch Konsensusempfehlungen veröffentlicht.

„Wir müssen so viele Strukturen wie möglich in die Rejuvenationsbehandlung einschließen und in jedem Drittel des Gesichts die Stütze wiederherstellen, straffen und revoluminisieren bzw. die mimische Muskulatur relaxieren“, erklärte Frau Dr. Tatjana Pavicic, niedergelassene Ästhetikexpertin in München. Den Konsensusempfehlungen zur kombinierten Intervention für die Restoration Beautification und Prävention von Alterserscheinungen zufolge, die am World Dermatology Congress in Dubai vorgestellt wurde, sollte als erster Schritt der mikrofokussierte Ultraschall zum
Einsatz kommen. Im Anschluss können Behandlungen mit Dermalfillern auf Basis von Hyaluronsäure (z. B. Belotero ${ }^{\circledast}$ ) und/oder Calciumhydroxylapatit (CaHA) am selben Tag in beliebiger Reihenfolge durchgeführt werden.

\section{Kollagensynthese in der tiefen Dermis anregen}

„Die am stärksten von Knochenatrophie betroffenen Areale entsprechen denen, die am stärksten und schnellsten altern", erklärte Frau Dr. Pavicic. Behandlungsziel müsse daher sein, diese tiefe Stütze des Gewebes wieder herzustellen. Durch die gezielte Energieabgabe von mikrofokussiertem Ultraschall wird die Kollagensynthese in der tiefen Dermis angeregt. „Es ist wirklich sinnvoll, mit dem in der Tiefe wirkendem fokussierten Ultraschall zu beginnen, dann mit einem Filler zu behandeln und schließlich die mimischen Falten mit Botulinumtoxin zu glätten“, so die Expertin. Dabei hat Incobotulinumtoxin (Bocouture ${ }^{\circledast}$ ) den Vorteil, als einziges BTX-Präparat für alle Falten des oberen Gesichts zugelassen zu sein, zudem muss es nicht im Kühlschrank gelagert werden.

Der ideale Volumenfiller für die Konturierung, Stimulation und Straffung ist ihrer Erfahrung zufolge CaHA, enthalten in Radiesse ${ }^{\circledast}$. Er sorgt für eine sofortige und langfristige Wirkung durch die Bildung neuer Hautfasern. Langfristig hat dieser Filler durch die Tatsache, dass er die Kollagenneogenese unterstützt auch verjüngungsfördernde Eigenschaften. Auch für die Konturierung ist dieser Filler besonders geeignet, da er im Gegensatz zu Hyaluronsäure keine hygroskopischen Effekte aufweist. Dr. Susanne Kammerer

Merz Expertentreff „Vom Profi zum Virtuosen“, Frankfurt am Main, 18. Februar 2017; Veranstalter: Merz 